\title{
Analysis and design of parallel-coupled high-gradient structure for ultrashort input power pulses
}

\author{
Yuliang Jiang $\odot$, Jiaru Shi, Hao Zha, Jiayang Liu $\odot$, Xiancai Lin, and Huaibi Chen \\ Department of Engineering Physics, Tsinghua University, Beijing CN-100084, China \\ and Key Laboratory of Particle and Radiation Imaging, Tsinghua University, Ministry of Education, \\ Beijing CN-100084, China
}

(Received 13 May 2021; accepted 25 October 2021; published 10 November 2021)

\begin{abstract}
Shortening the pulse length of input power is a possible approach to raise the accelerating gradient limit of normal conducting structures. We present a novel design of a parallel-coupled structure that operates at ultrashort input power pulses. The principle of the design is to shorten the filling time of the whole structure by feeding the cells individually so that the duration of power transmission between cells can be saved. An X-band (11.994 GHz) parallel-coupled structure design with a $10 \mathrm{~ns}$ pulse length is presented in this work. Previous high-power tests of X-band structure prototypes show that a gradient of $120 \mathrm{MV} / \mathrm{m}$ could be achieved for a $200 \mathrm{~ns}$ pulse length. Based on the law requiring that $E^{30} t^{5}=$ constant, this 10-ns-structure design should be able to reach a gradient of $200 \mathrm{MV} / \mathrm{m}$. A detailed circuit model and real-time electromagnetic field simulation methods for designing the parallel-coupling structure are also presented and discussed in this paper.
\end{abstract}

DOI: 10.1103/PhysRevAccelBeams.24.112002

\section{INTRODUCTION}

High-gradient normal conducting structures have been studied for several decades as promising candidates for linear accelerator projects such as the compact linear collider (CLIC) and free-electron lasers [1-7]. The main factor affecting the accelerating gradient is rf breakdown. The accelerating structure's performance at a given gradient and pulse length is characterized by the breakdown rate (BDR) [8-11]. Although the mechanism of rf breakdown has not been satisfactorily explained [12-16], an empirical equation has been given to estimate BDR dependencies on the gradient and pulse length based on data collected from a CLIC breakdown study [17]:

$$
\mathrm{BDR} \propto E^{30} t^{5}
$$

where $E$ is the accelerating gradient and $t$ is the input pulse length.

This equation indicates that a higher accelerating gradient may be achievable with a shorter pulse length. To date, the undamped design of CLIC X-band traveling-wave (TW) structures named T24 has achieved a gradient of

\footnotetext{
*zha_hao@mail.tsinghua.edu.cn
}

Published by the American Physical Society under the terms of the Creative Commons Attribution 4.0 International license. Further distribution of this work must maintain attribution to the author(s) and the published article's title, journal citation, and DOI.
$120 \mathrm{MV} / \mathrm{m}$ with a pulse length of $200 \mathrm{~ns}$ [18]. According to Eq. (1), the scaled gradient may be $200 \mathrm{MV} / \mathrm{m}$ for a similar design if the pulse length could be reduced to $10 \mathrm{~ns}$.

However, the applicability of the scaling law at the 10-ns scale is still in doubt since the minimum pulse length of the fitting data is $40 \mathrm{~ns}[17,19]$. Moreover, the available experimental data based on the ultrashort-pulse regime were obtained on single cavities or at a higher frequency and these results have shown various results regarding gradients. On the one hand, earlier 30-GHz TW structures tested at the beam-driven CLIC test facilities (CTF-II) have a shorter filling time than that of the $\mathrm{X}$-band. Accelerating gradients of 140,100 , and $70 \mathrm{MV} / \mathrm{m}$ were achieved with pulse lengths of 4,8 , and $16 \mathrm{~ns}$, respectively [20]. The CTF-II results, together with the X-band results, also show that the obtainable surface fields have a weak frequency dependence between 11 and $39 \mathrm{GHz}$, which favors direct gradient comparison of structures within this range $[20,21]$. The achieved gradients are lower than the estimated gradient scaled from Eq. (1). On the other hand, a single beam-driven X-band TW cavity recently tested on Argonne achieved a gradient of $310 \mathrm{MV} / \mathrm{m}$ with a pulse full-width at half-maximum of $6 \mathrm{~ns}$ [22], the gradient of which is even higher than the scaled one. The gradient difference of the aforementioned tests may result from different design and rf parameters such as structure geometries, structure length (multi/single), and power sources [23]. Thus, designing a CLIC-type, X-band, multicell structure for ultrashort pulses should bridge the gap and offer insight into the underlying mechanism. 
TABLE I. Parameters of CLIC-T24 and the modified design with a filling time of $10 \mathrm{~ns}$ at a gradient of $120 \mathrm{MV} / \mathrm{m}$.

\begin{tabular}{lll}
\hline \hline Parameter & CLIC-T24-nominal & CLIC-T24-10 ns \\
\hline Filling time & $56 \mathrm{~ns}$ & $10 \mathrm{~ns}$ \\
Iris aperture (first/last) & $3.15 / 2.35 \mathrm{~mm}$ & $4.85 / 4.52 \mathrm{~mm}$ \\
Input power & $57 \mathrm{MW}$ & $400 \mathrm{MW}$ \\
Maximum electric field & $257 \mathrm{MV} / \mathrm{m}$ & $340 \mathrm{MV} / \mathrm{m}$ \\
Maximum $S c^{\mathrm{a}}$ & $5 \mathrm{MW} / \mathrm{mm}^{2}$ & $17.9 \mathrm{MW} / \mathrm{mm}^{2}$ \\
Shunt impedance & $123 \mathrm{M} \Omega / \mathrm{m}$ & $81 \mathrm{M} \Omega / \mathrm{m}$ \\
\hline \hline
\end{tabular}

${ }^{\mathrm{a}} \mathrm{S} c$ is the modified Poynting vector.

For most of the linear accelerating structures, including both TW and standing-wave (SW) designs, rf power is fed through one input coupler and transmitted over cells. The filling time of these X-band structures is usually much longer than $10 \mathrm{~ns}[16,24]$. Increasing the iris aperture can reduce the filling time but will result in a decline in the shunt impedance and an increase in the surface field. The estimated parameters of this modified CLIC-T24 design as well as of the nominal design are shown in Table I, which seem less attractive for practical use.

Another approach to shortening the filling time is to reduce the number of cells per input coupler. The most thorough solution is to power cells in parallel rather than in series, thus reducing the time spent on power transmission over cells. This SW structure with a parallel power supply to cells was proposed in the 1970s [25] and similar concepts have been investigated in several cases [26-28]. Since the power coupling between neighboring cells is avoided, the parallel structure can prevent severe rf breakdowns near the beam aperture and has more design space to improve the performance in terms of, e.g., the shunt impedance [29]. Previous studies usually focused on these structural advantages [26-28]. Moreover, traditional multicell structures, including both parallel-coupled ones and series-coupled ones, are designed with a typically long pulse length of more than $100 \mathrm{~ns}[18,28]$. A multicell parallel-coupled structure should also be able to work at ultrashort pulses after dedicated analysis, which may result in a significant increase in the gradient limit.

This work studies a conceptual parallel-coupled highgradient structure for ultrashort input power pulses. In Sec. II, a circuit model is developed and analyzed to calculate the coupling coefficient of the whole structure from all components. In Sec. III, a preliminary X-band parallel-coupled structure design using the model presented in Sec. II is introduced. Section IV develops a method for calculating the real-time electromagnetic fields. Thus, the process of power filling in the structure during the ultrashort time could be visually simulated and verified. According to the calculation, significant advantages are presented for the parallel-coupled structure operating at ultrashort pulses, and the search for a high gradient is shown to be promising.
Parallel coupled structure

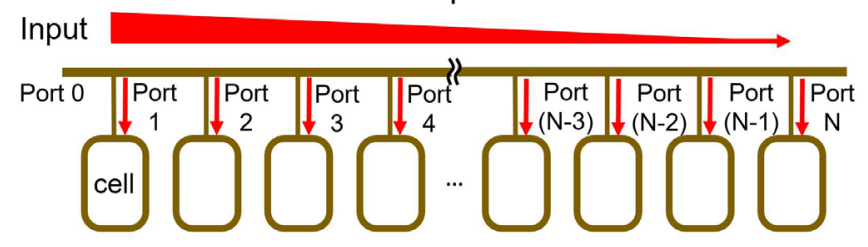

FIG. 1. Scheme of the parallel-coupled accelerating structure.

\section{SYSTEM ANALYSIS}

A conceptual drawing of the parallel-coupled structure, which consists of a parallel rf feed system and individual cells, is shown in Fig. 1. The input power is fed into $\mathrm{N}$ isolated cells through tap-off ports. A simplified case was studied to analyze only the power at the input port, with the assumption of a matched load at the end of each tapoff port [28]. To analyze the power flow among the total $(\mathrm{N}+1)$ ports, a more general expression of the microwave network should be derived. Here, elements of the scattering matrix are defined as $s_{n m}$. The subscript $n$ or $m$ indicates the port: 0 for the input port and $1-\mathrm{N}$ for tap-off ports to cells.

An essential issue of the coupling line is equal power division, where all identical cells receive the same field amplitude to avoid a 'hot' cell in terms of rf breakdown. The parallel rf feed system could be disassembled to multiple T-junctions, with each junction feeding one cell, as shown in Fig. 2. For T-junctions with different geometries, the scattering matrices should be customized separately and then cascaded section by section. A simpler design is to adopt identical T-junctions so that the network parameters of the whole parallel rf feed system could be derived directly.

The T-junction is equivalent to a three-port network, as shown in the inset of Fig. 2. It is assumed to be an E-plane component; thus, Ports (1) and (2) receive opposite signals scattered from Port (3). Considering that the ideal T-junction is reciprocal and lossless, the general scattering matrix could be described as follows: [28]

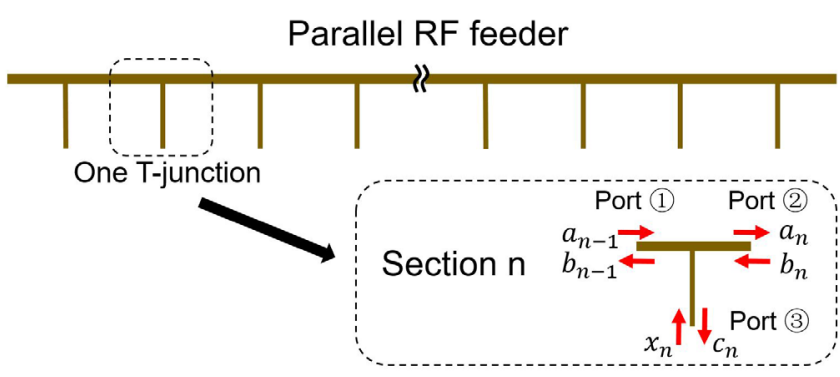

FIG. 2. Cascading of identical T-junctions. The inset in the lower right is the equivalent microwave network of the $n$th T-junction. 


$$
S=\frac{1}{2}\left[\begin{array}{ccc}
e^{j \theta}-u & e^{j \theta}+u & v \\
e^{j \theta}+u & e^{j \theta}-u & -v \\
v & -v & \frac{2 u^{*} v}{v^{*}}
\end{array}\right]
$$

where $u$ and $v$ are undetermined complex numbers, $u^{*}$ and $v^{*}$ are their conjugate complex numbers, respectively, and $|u|^{2}+|v|^{2} / 2=1$. $\theta$ is an undetermined degree number.

\section{A. Incident power from the input port}

In Fig. 2, $a_{n}$ and $b_{n}$ denote the amplitudes of the front wave and the back wave after the $n$th section. When there is no incident signal from any tap-off port, the three-port network can be expressed as

$$
\begin{gathered}
\frac{1}{2}\left[\begin{array}{ccc}
e^{j \theta}-u & e^{j \theta}+u & v \\
e^{j \theta}+u & e^{j \theta}-u & -v \\
v & -v & \frac{2 u^{*} v}{v^{*}}
\end{array}\right]\left[\begin{array}{c}
a_{n-1} \\
b_{n} \\
0
\end{array}\right]=\left[\begin{array}{c}
b_{n-1} \\
a_{n} \\
c_{n}
\end{array}\right], \\
{\left[\begin{array}{c}
a_{n} \\
b_{n}
\end{array}\right]=\frac{1}{e^{j \theta}+u}\left[\begin{array}{cc}
2 u e^{j \theta} & e^{j \theta}-u \\
u-e^{j \theta} & 2
\end{array}\right]\left[\begin{array}{c}
a_{n-1} \\
b_{n-1}
\end{array}\right],}
\end{gathered}
$$

where $c_{n}$ is the output of the $n$th tap-off port and is calculated as

$c_{n}=\frac{v\left(a_{n}-e^{j \theta} b_{n}\right)}{e^{j \theta}+u} \quad$ and $\quad c_{n+1}=\frac{v\left(e^{j \theta} a_{n}-b_{n}\right)}{e^{j \theta}+u}$.

To ensure equal power division, $\left|c_{n}\right|=\left|c_{n+1}\right|$ holds for all integer values of $n$. Considering that $a_{n}$ and $b_{n}$ vary with $n, e^{j \theta}= \pm 1$ should be satisfied. The choice of $e^{j \theta}$ influences only the sign of $c_{n}$. The discussion below focuses on the case of $e^{j \theta}=1$, as the preliminary rf design in Sec. III is based on this case. Therefore, the transmission matrix from $\left(a_{n-1}, b_{n-1}\right)$ to $\left(a_{n}, b_{n}\right)$ is transformed as

$$
\begin{aligned}
{\left[\begin{array}{l}
a_{n} \\
b_{n}
\end{array}\right] } & =\frac{1}{1+u}\left[\begin{array}{cc}
2 u & 1-u \\
u-1 & 2
\end{array}\right]\left[\begin{array}{l}
a_{n-1} \\
b_{n-1}
\end{array}\right] \\
& =\left(\left[\begin{array}{ll}
1 & 0 \\
0 & 1
\end{array}\right]+\rho\left[\begin{array}{ll}
-1 & 1 \\
-1 & 1
\end{array}\right]\right)\left[\begin{array}{l}
a_{n-1} \\
b_{n-1}
\end{array}\right] \\
& =(\mathbf{A}+\rho \mathbf{B})\left[\begin{array}{l}
a_{n-1} \\
b_{n-1}
\end{array}\right], \\
{\left[\begin{array}{l}
a_{n} \\
b_{n}
\end{array}\right] } & =\left(\left[\begin{array}{ll}
1 & 0 \\
0 & 1
\end{array}\right]+n \rho\left[\begin{array}{ll}
-1 & 1 \\
-1 & 1
\end{array}\right]\right)\left[\begin{array}{l}
a_{0} \\
b_{0}
\end{array}\right],
\end{aligned}
$$

where $\rho=(1-u) /(1+u), a_{0}$ and $b_{0}$ are the incident and reflected waves at the input port, respectively. The second matrix term satisfies $\mathbf{B}^{2}=\mathbf{0}$, which simplifies the calculation of the $n$th power of the transmission matrix, as shown in Eq. (7).
Since the parallel $\mathrm{rf}$ feed system is terminated by a short end, the reflection at Port (2) of the last section $N$ is -1 , which gives $a_{N}+b_{N}=0$. Derived from Eq. (7), we have $a_{0}+b_{0}=2 N \rho\left(a_{0}-b_{0}\right)$. Thus, the reflection coefficient $s_{00}$ could be calculated from $b_{0} / a_{0}$ and the coefficients of power division $s_{0 n}$ are equal to $c_{n} / a_{0}$, as shown in Eq. (8) below.

$$
s_{00}=\frac{b_{0}}{a_{0}}=\frac{2 N \rho-1}{2 N \rho+1}, \quad s_{0 n}=\frac{c_{n}}{a_{0}}=\frac{v(\rho+1)}{2 N \rho+1}
$$

\section{B. Incident power from the tap-off port}

Matrix elements $s_{m n}$ for $m \neq 0$ are solved in another case where the incident signal $x$ is from the $m$ th tap-off port. Then, the equation for $\left(a_{m}, b_{m}\right)$ in this section should be modified as

$$
\frac{1}{2}\left[\begin{array}{ccc}
1-u & 1+u & v \\
1+u & 1-u & -v \\
v & -v & \frac{2 u^{*} v}{v^{*}}
\end{array}\right]\left[\begin{array}{c}
a_{m-1} \\
b_{m} \\
x
\end{array}\right]=\left[\begin{array}{c}
b_{m-1} \\
a_{m} \\
c_{m}
\end{array}\right] .
$$

Except for the $m$ th section with an incident signal $x$, Eq. (6) is applicable to the other sections under an additional condition, i.e., $a_{0}=0$. Thus, $b_{0}=x s_{m 0}=x s_{0 m}=$ $x v(\rho+1) /(2 N \rho+1)$ is the signal scattered from Ports $m$ to 0 . The values of $\left(a_{n}, b_{n}\right)$ are given as

$$
\left[\begin{array}{l}
a_{n} \\
b_{n}
\end{array}\right]= \begin{cases}b_{0}\left[\begin{array}{c}
n \rho \\
1+n \rho
\end{array}\right], & n<m \\
b_{0}\left[\begin{array}{c}
n \rho \\
1+n \rho
\end{array}\right]-\frac{v x}{2}\left[\begin{array}{c}
\rho+1 \\
\rho+1
\end{array}\right], & n \geq m\end{cases}
$$

The output signals $c_{n}$ of the tap-off ports can also be calculated from Eqs. (3) and (7) as

$$
\begin{gathered}
c_{n}=\frac{v\left(a_{n}-b_{n}\right)}{1+u}-\frac{v b_{0}}{1+u}, \quad n \neq m \quad \text { and } \\
c_{m}=\frac{v\left(a_{m-1}-b_{m}\right)}{1+u}+\frac{u^{*} v x}{v^{*}}, \quad n=m .
\end{gathered}
$$

This gives matrix elements $s_{n m}=c_{n} / x$ and $s_{m m}=c_{m} / x$. Thus, the scattering matrix of the parallel $\mathrm{rf}$ feed system is solved, and all elements are listed in Eqs. (8) and (12).

$s_{n m}=-\frac{1}{2} \frac{v^{2}(\rho+1)^{2}}{2 N \rho+1} \quad$ and $\quad s_{m m}=s_{n m}+\frac{v}{v^{*}} \frac{1+u^{*}}{1+u}$ 


\section{Scattering matrix with unmatched cells}

The scattering matrix of the parallel-feeding network is solved under the assumption that all ports are matched. However, the structure cell may reflect rf power if it is not critically coupled. This reflected power will flow back into the network and affect other cells. To study this effect, an equation of the whole system is established as

$$
\left[\begin{array}{cccc}
s_{00} & s_{01} & \cdots & s_{0 N} \\
s_{10} & s_{11} & \cdots & s_{1 N} \\
\vdots & \vdots & \ddots & \vdots \\
s_{N 0} & s_{N 1} & \cdots & s_{N N}
\end{array}\right]\left[\begin{array}{c}
a_{0} \\
x_{1} \\
\vdots \\
x_{N}
\end{array}\right]=\left[\begin{array}{c}
b_{0} \\
c_{1} \\
\vdots \\
c_{N}
\end{array}\right],
$$

where $a_{0}$ is the amplitude of the input signal, $b_{0}$ is the reflected one, and $c_{n}$ and $x_{n}$ represent the incident signal and the reflected signal of the $n$th cell, respectively.

According to the circuit model of a resonant cavity, the power transmitted to the cavity $g_{n}=c_{n}-x_{n}$ is proportional to the field amplitude of the cell and varies during the filling process. A differential equation is derived based on the circuit model to study the dynamics of power filling and reflection:

$$
\frac{d g_{n}}{d t}=\frac{\omega}{2 Q_{0}}\left[2 \beta c_{n}-(\beta+1) g_{n}\right],
$$

where $\omega, Q_{0}$, and $\beta$ are the angular resonant frequency, unloaded quality factor, and coupling coefficient of the single cell, respectively. Let $\mathbf{g}=\left[g_{1} \cdots g_{N}\right]^{T}$ and $\mathbf{c}=$ $\left[c_{1} \cdots c_{N}\right]$ for all cells. With these equations, together with Eqs. (13) and (14), the differential equation could be extended to the following vector form:

$$
\begin{gathered}
\frac{d \mathbf{g}}{d t}=\frac{\omega}{2 Q_{0}}\left(2 \beta a_{0} \mathbf{M}\left[\begin{array}{c}
s_{10} \\
\vdots \\
s_{N 0}
\end{array}\right]+(\beta-1) \mathbf{g}-2 \beta \mathbf{M g}\right), \\
\mathbf{M}=\left[\begin{array}{ccc}
1-s_{11} & \cdots & -s_{1 N} \\
\vdots & \ddots & \vdots \\
-s_{N 1} & \cdots & 1-s_{N N}
\end{array}\right]^{-1} .
\end{gathered}
$$

The values of $s_{n 0}, s_{n m}$, and $s_{m m}$ are constants over any $n$ and $m$. Thus, a trivial eigenvector of Eq. (13) is $\left[\begin{array}{lll}1 & \ldots & 1\end{array}\right]^{T}$. This eigenvector is regarded as a solution of an even field distribution over cells, which is the one we want to study. The $(\mathrm{N}+1)$-dimensional differential equations could be degenerated to a single-dimensional one for this eigenvector, as shown in Eq. 17(a). The matrices are replaced with the corresponding eigenvalue, which can be expressed as shown in Eq. 17(c).

$$
\begin{aligned}
\frac{d g}{d t} & =\frac{\omega}{2 Q_{0}}\left[\frac{d}{1-\lambda_{p}}-\left(1+\beta \frac{1+\lambda_{p}}{1-\lambda_{p}}\right) g\right], \\
d & =2 \beta a_{0} s_{10}=\frac{2 \beta v(\rho+1)}{2 N \rho+1} a_{0}, \\
\lambda_{p} & =\sum_{n=1}^{N} s_{n m}=\frac{1-2 N \rho^{*}}{1+2 N \rho} \frac{1+\rho}{1+\rho^{*}} \frac{v}{v^{*}} .
\end{aligned}
$$

Equation 17(a) describes the dynamic of building up the field in the cells of the parallel-coupled structure. It is similar to the filling equation of a conventional SW structure. Thus, the equivalent coupling coefficient $\left(\beta^{\prime}\right)$ of the full structure is $\beta^{\prime}=\beta\left(1+\lambda_{p}\right)\left(1-\lambda_{p}\right)$.

To avoid phase shifting, the coupling coefficient should not have an imaginary part. A simple case is one in which $\rho$ is real and $v / v^{*}= \pm 1$. In this case, the solution to Eq. 17(a) and the equivalent coupling coefficient $\left(\beta^{\prime}\right)$ are expressed as

$$
\begin{aligned}
g(t) & =\frac{2 a_{0} \sqrt{\beta \beta^{\prime} / N}}{1+\beta^{\prime}}\left(1-e^{-\frac{\omega\left(1+\beta^{\prime}\right)}{2 Q_{0}} t}\right) \quad \text { and } \\
\beta^{\prime} & =(2 N \rho)^{\mp 1} \beta .
\end{aligned}
$$

At this point, the analytical power-filling process of a parallel-coupled structure is derived. Because all cells are powered simultaneously rather than sequentially, a highgradient accelerating field can be built up quickly under the overcoupled condition $\beta^{\prime} \gg 1$. Since the increase in the field amplitude occurs at a gradually lower rate over time, the input pulse length is optimized to have the maximum filling efficiency, which is defined as the ratio of the structure's stored to emitted energy of the input pulse. Numerical calculations show that the maximum filling efficiency is approximately $0.815 \beta^{\prime} /\left(1+\beta^{\prime}\right)$, corresponding to a optimum pulse length $t_{f} \simeq 2.5 Q_{0} / \omega\left(1+\beta^{\prime}\right)$, as shown in Fig. 3.

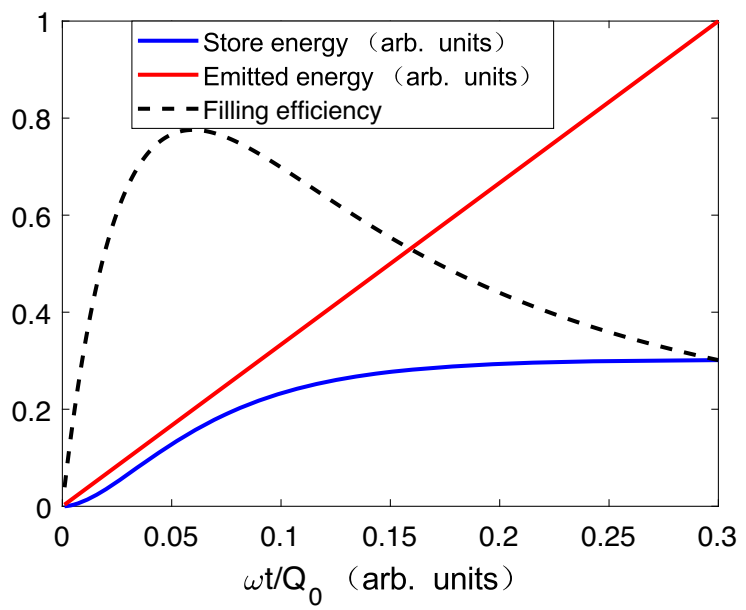

FIG. 3. Stored energy vs filling time in the cavity cell (numbers were calcualted at $\beta^{\prime}=20$ ). 


\section{PRELIMINARY rf DESIGN}

To verify the above analysis and compare with traditional TW and SW structures, an X-band parallel-coupled structure was designed preliminarily. Different from previous critically coupled parallel structures, this design should be overcoupled to achieve fast power filling. The reflected power of these two identical structures with the proper distance can be directed away from the power source through a 3-dB hybrid. According to Eq. 18(b), the structure's coupling coefficient $\left(\beta^{\prime}\right)$ is related to $S$-parameters of the T-junction, the cell's coupling coefficient $(\beta)$, and the number $(N)$ of tap-off ports.

Equal power division is a prerequisite for the structure design. The condition that $e^{j \theta}= \pm 1$ corresponds to a phase advance of 0 or $\pi$ between two neighboring tap-off ports. Choosing a phase advance of $\pi$ per cell can simplify the design rather than complicating the tap-off ports with varied length. Moreover, cells operating at the $\pi$-mode can be regarded as isolated from each other theoretically [28], which matches our assumption. Thus, the structure was designed to operate at the $\pi$ mode.

\section{A. rf design of the single cell}

In the $\pi$ mode, a $12 \mathrm{GHz}$ cell has a length of $\lambda / 2=$ $12.5 \mathrm{~mm}$, where $\lambda$ is the free-space wavelength. The cell geometries are based on the middle cell of CLIC-T24, with an elliptical $2.0 \mathrm{~mm}$ thick iris and an aperture radius of $2.75 \mathrm{~mm}$, as shown in Fig. 4. The practical coupling coefficient between neighboring cells is estimated as $k \sim a^{3} e^{-\alpha_{e} d}$, where $2 a$ is the iris aperture and $d$ is the thickness of the diaphragm [30]. Even though there may still exist rf power coupling between two consecutive cells in practice, this intercross has very little effect on the equations in the case of a small iris aperture [28]. The rf parameters of the cell structure are listed in Table II, together with the middle cell design of the nominal CLIC-T24.

The cylindrical cell is side-coupled through a rectangular waveguide and the coupling coefficient $(\beta)$ could be
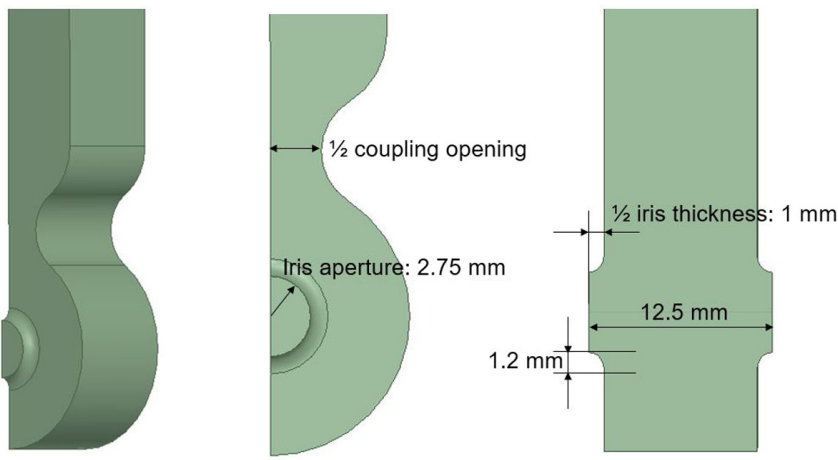

FIG. 4. rf design of the accelerating cell.
TABLE II. rf parameters of the parallel-feeding cell and the middle cell of CLIC-T24 at a gradient of $200 \mathrm{MV} / \mathrm{m}$.

\begin{tabular}{lcc}
\hline \hline $\begin{array}{l}\text { rf parameters } \\
\text { at } 200 \mathrm{MV} / \mathrm{m}\end{array}$ & $\begin{array}{c}\text { Parallel-feeding } \\
\text { cell }\end{array}$ & $\begin{array}{c}\text { CLIC-T24 } \\
\text { middle cell }\end{array}$ \\
\hline$Q_{0}$ & 8173 & 6898 \\
$R / Q(\Omega / \mathrm{m})$ & 12000 & 17790 \\
Stored energy $(\mathrm{J})$ & 0.55 & 0.25 \\
Maximum $E$-field $(\mathrm{MV} / \mathrm{m})$ & 257 & 340 \\
Maximum $H$-field $(\mathrm{kA} / \mathrm{m})$ & 840 & 490 \\
Maximum $S c\left(\mathrm{MW} / \mathrm{mm}^{2}\right)$ & 12.72 & 13.48 \\
\hline \hline
\end{tabular}

modified by adjusting the coupling opening's width. The enhanced surface-field patterns due to side-coupling are shown in Fig. 5, where the magnetic field is higher near the coupling opening. However, the peak modified Poynting vector $\left(S_{c}\right)$, with which the breakdown damage is more correlated, is still near the beam aperture. Moreover, previous test of a single-feed cell with side-coupling did not show a higher BDR due to the increased magnetic field, in contrast to the case of a dual-feed cell with side-coupling [31]. Thus, the single-feed, side-coupled cell is convincing for the high-gradient parallel-coupled structure.

\section{B. rf design of the parallel waveguide feeder}

Rectangular waveguides were adopted as the parallel rf feeder for high-power transmission. To have more space for the waveguide coupler at the tap-off port and more symmetric geometry, an alternating single-feed scheme based on two transmission waveguides was adopted, as shown as Fig. 6. Therefore, the tap-off port for each branch has the same phase $\left(e^{j \theta}=1\right)$ and Eq. 18(b) can be further expressed as
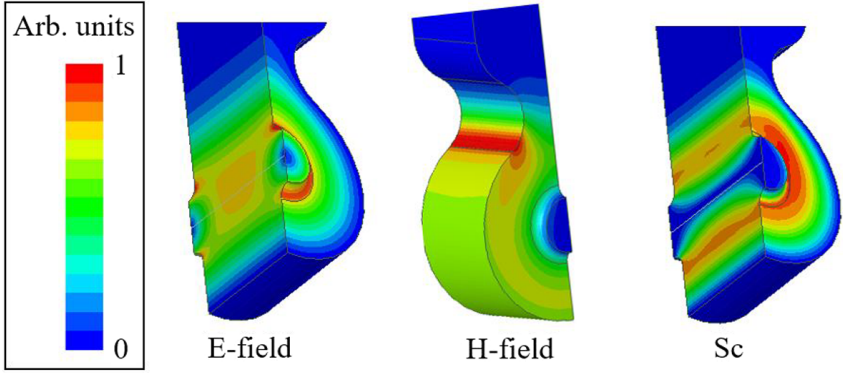

FIG. 5. Surface field patterns of the accelerating cell.

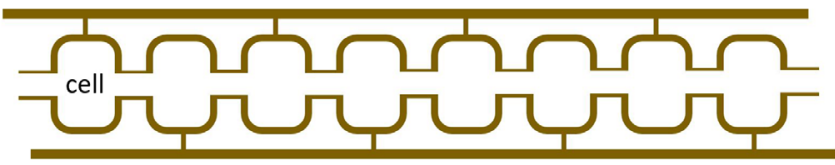

FIG. 6. Scheme of parallel-coupled structure with two rectangular feeding waveguides. 


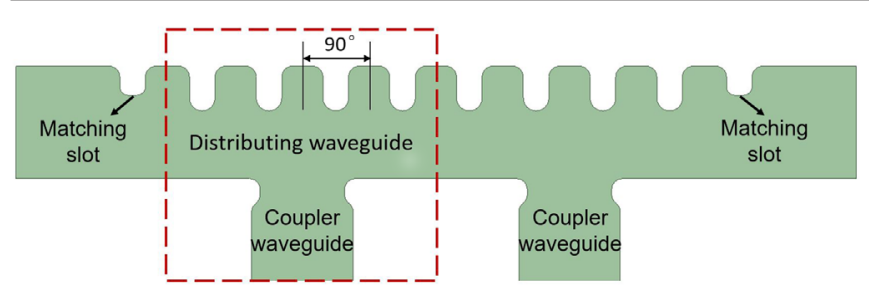

FIG. 7. rf design of a parallel waveguide feeder.

$$
\beta^{\prime}=2 N \rho \beta=2 N \frac{1-\left|S_{33}\right|}{1+\left|S_{33}\right|} \beta,
$$

where $\left|S_{33}\right|$ is the reflection amplitude of the tap-off port of a T-junction.

Phase synchronization of the rf power during transmission is also essential for power division. The rf phase velocity was reduced to the speed of light by introducing the slow-wave structure of periodic slots, as shown in Fig. 7. The transmission part was designed with four corrugated slots per T-junction, which means an approximately $\pi / 2$ phase shift for each corrugation period. The coupler waveguide was then connected to the transmission part through a transition structure. The phase difference between $S_{11}$ and $S_{12}$ introduced by the coupler waveguide was also corrected by modifying this transition part. The scattering matrix of a valid T-junction can be expressed as

$$
S_{3}=\left[\begin{array}{ccc}
0.216 & 0.784 & 0.582 j \\
0.784 & 0.216 & -0.582 j \\
0.582 j & -0.582 j & -0.569
\end{array}\right] \text {. }
$$

To match with smooth waveguides, additional slots were optimized and added to the ends of the corrugated transmission waveguide, as shown in Fig. 7. Both the slowwave slots and the matching slots led to an intense local E-field enhancement, which is a potential limitation for high-gradient operation of a multicell structure.

Thus, the equivalent coupling coefficient of the whole structure can be expressed as $\beta^{\prime}=2 N \rho \beta=$ $2 N \beta\left(1-\left|S_{33}\right|\right) /\left(1+\left|S_{33}\right|\right) \simeq 0.55 N \beta$, where $N$ is the T-junction number of a parallel waveguide feeder.

\section{C. rf design of the whole parallel-coupled structure}

The whole structure was then designed, consisting of 16 accelerating cells and two parallel waveguide feeders, as shown in Fig. 8. Due to developments of the split structure [32], the cells and the parallel waveguide feeders can be made of two halves instead of many individual parts [28], especially for structures with a simple topology. The number of cells was chosen to match the length of CLIC-T24 $(16 \pi=24 * 2 \pi / 3)$. Substituting the designed rf parameters into Eq. (19), the structure's coupling coefficient is estimated as $\beta^{\prime} \simeq 4.4 \beta$, which was verified by simulating the whole structure directly through CST [33].

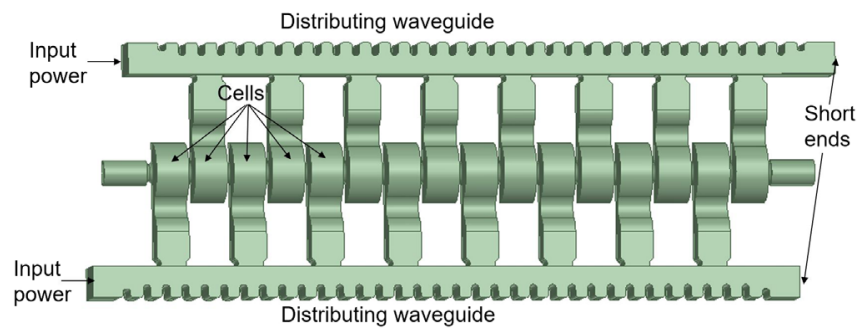

FIG. 8. rf design of the whole parallel-coupled structure.

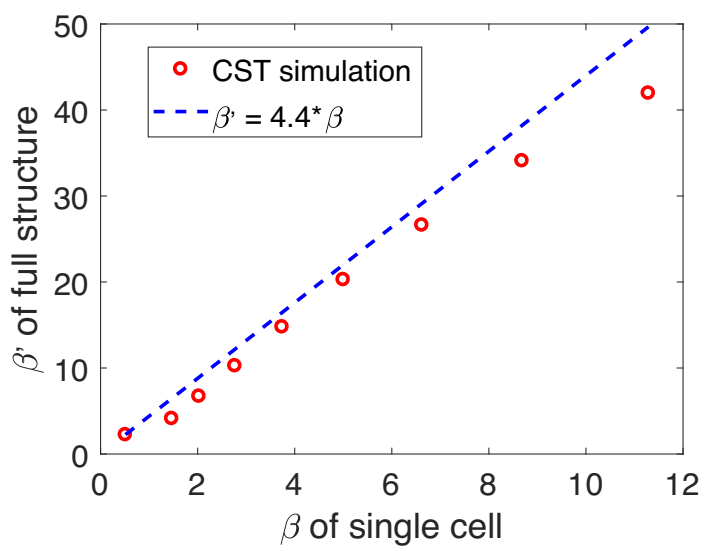

FIG. 9. Coupling coefficients $\left(\beta^{\prime}\right)$ of the whole structure with different single-cell coupling coefficients $(\beta)$.

The coupling coefficients of a single cell $(\beta)$ and the structure $\left(\beta^{\prime}\right)$ vary with the width of the coupling opening, and their values can be calculated from the simulated $S$-parameters. The analytical and simulated relationships between $\beta$ and $\beta^{\prime}$ of the parallel-coupled structure are shown in Fig. 9, which validate the theoretical analysis. The slight difference between the simulation and the derived calculation may result from the end cells, since their cavity radii were modified due to the use of a different boundary condition.

For $10 \mathrm{~ns}$ ultrashort input pulses, the optimized coupling coefficients are approximately $\beta^{\prime} \simeq 26$ and $\beta \simeq 5.9$. The $S$-parameter of the parallel-coupled structure is shown in Fig. 10.

\section{REAL-TIME ELECTROMAGNETIC FIELD CALCULATION}

The simulation conducted in Sec. III calculated only the steady-state field in the structure. However, a pulse length of $10 \mathrm{~ns}$ is far too low to approach the steady state. To verify the expected filling process, a real-time electromagnetic field calculation is needed. Although the CST code implements the real-time module, a considerable computational cost in terms of time and hardware is required to obtain accurate results.

In this section, a method based on the fast Fourier transform (FFT) was applied to obtain the real-time fields 


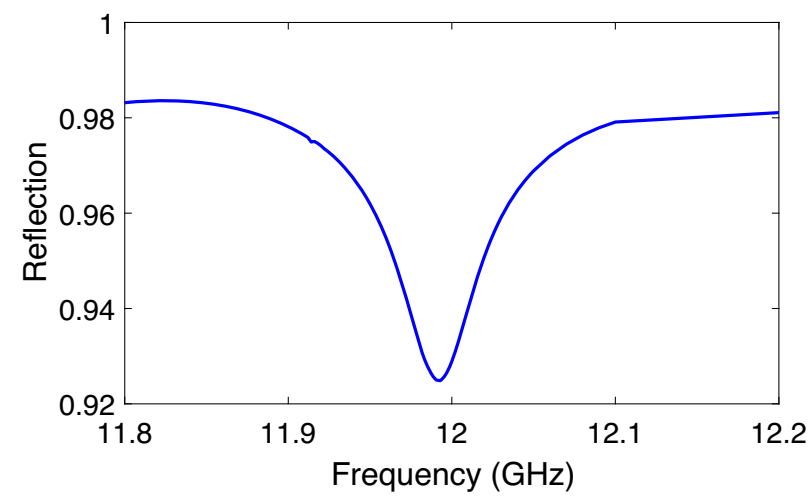

FIG. 10. Reflection of the parallel-coupled structure.

with an arbitrary input pulse shape. The frequency-domain data were gained by calculating the field at multiple frequencies. The real-time fields at a grid point could be calculated by performing an inverse FFT on the product of the frequency-domain field data and spectrum of the input pulse. To achieve a sub-ns resolution in the time domain, such as $0.1 \mathrm{~ns}$, the field data over a broad frequency range of $10 \mathrm{GHz}$ are needed. To reduce the required computation time and data size, the simulation was carried out at unequal frequency intervals with fine steps near the operating frequency only. The full frequency-domain data used in the FFT were calculated by interpolation of the simulated data. This method was applied to all grids to generate the real-time field plot of the structure. After obtaining the field data from the CST simulation, all calculations, including the field plot generation, could be conducted in the MATLAB code.

Figure 11 shows the real-time calculation of the longitudinal E-field $E_{z}$ using the above method on a CLIC-T24 structure with a 60-ns input pulse. The animation demonstrates consecutive building up of the field over cells, and the filling time estimated in the animation agrees with the results in Table I.

The real-time accelerating field of the designed parallelcoupled structure was calculated with an input pulse of

(a) Time $=15 \mathrm{~ns}$ ए?

(b) Time $=30 \mathrm{~ns}$ Pen?

(c) Time $=45 \mathrm{~ns}$

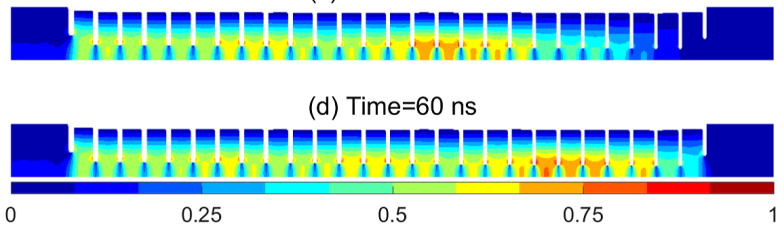

FIG. 11. Normalized temporal $E_{z}$ distribution of a CLIC-T24 structure with a 60 -ns input pulse.

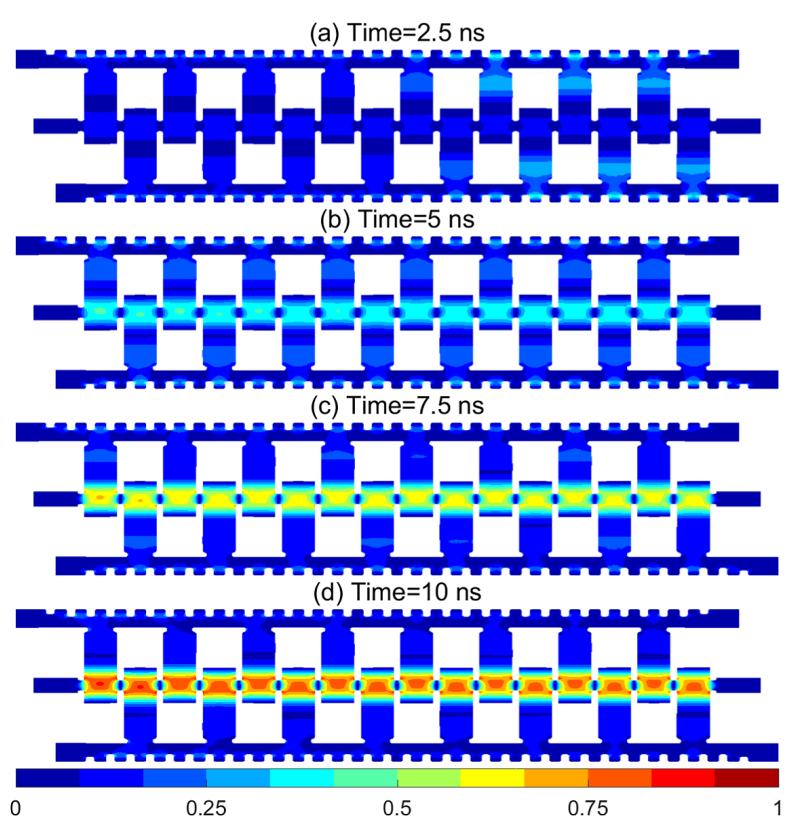

FIG. 12. Normalized temporal $E_{z}$ distribution of the designed parallel-coupled structure with a 1.1-GW, 10-ns input pulse.

1.1 GW power and $10 \mathrm{~ns}$ pulse length, as shown in Fig. 12. The rising time of the pulse was set to be 1 ns. The accelerating gradient over the cells retained an even distribution during the filling process, and the calculated field distribution at $10 \mathrm{~ns}$ is shown in Fig. 13. The average gradient reached its maximum value of $200 \mathrm{MV} / \mathrm{m}$ at the end of the 10-ns pulse, which agrees with the calculation in Sec. III. The animation shows that a minimum time of $4 \mathrm{~ns}$ was required to build the even distribution of the accelerating field. This is the lowest limit of the pulse length for the parallel-coupled structures at this length.

To gain a higher peak power from commercial power sources such as klystrons, an rf pulse compressor could be used [34-38], and the cascaded pulse-compression concept has been demonstrated at the Tsinghua S-band test facility [39]. Based on the parameters of the X-band test stands at Tsinghua University, an X-band two-stage rf compressor is under development. Since the parallelcoupled structure presented in this work is designed for the single-bunch operation, the unflat pulse still works.

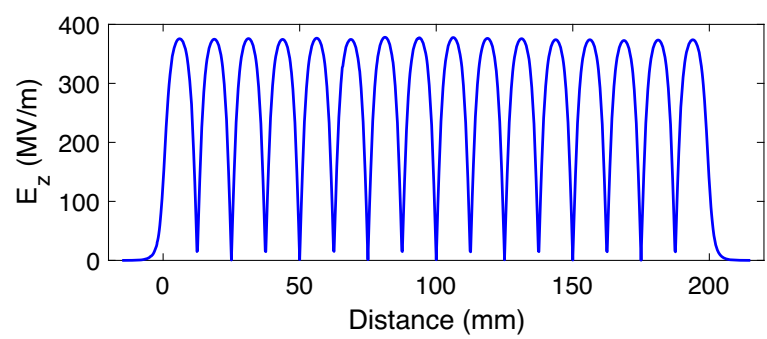

FIG. 13. $E_{z}$ field distribution on the axis of the designed parallel-coupled structure at $10 \mathrm{~ns}$. 


\section{CONCLUSIONS}

A novel parallel-coupled structure is introduced in this work. The structure uses a distributed coupling network to achieve ultrafast power filling in each cell. A circuit model of the distributed coupling is discussed and used to design the short-filling structure. This analysis can be further applied to other specific structures or researches on system responses of a parallel-coupled structure under different conditions. The real-time electromagnetic field calculation is also developed to observe and verify the filling process of the accelerating structure.

An X-band parallel-coupled structure design is demonstrated. It has 16 cells, and the length of the structure is $20 \mathrm{~cm}$, which is comparable to the X-band structure design of the CLIC main linac. The filling time of the parallelcoupled structure is only $10 \mathrm{~ns}$; thus, $200 \mathrm{MV} / \mathrm{m}$ is achievable according to the test data of CLIC structures and the law requiring that $E^{30} t^{5}=$ constant.

Although promising parameters are obtained, the presented structure is still a preliminary design aimed at verifying the principle of achieving an ultrashort filling time. Future prototypes may be developed by using longitude split halves, which are easier to fabricate. The slow-wave geometry in the coupling line should be redesigned to reduce the surface field. High-power testing on the parallel-coupled structure prototypes will also be performed.

\section{ACKNOWLEDGMENTS}

The authors thank Jiahang Shao and Alexej Grudiev for the fruitful discussions. This work was supported by the National Natural Science Foundation of China (NSFC), Grant No. 11975139.

[1] CLIC, Updated baseline for a staged compact linear collider, arXiv:1608.07537.

[2] T. Inagaki, C. Kondo, H. Maesaka, T. Ohshima, Y. Otake, T. Sakurai, K. Shirasawa, and T. Shintake, High-gradient $c$-band linac for a compact X-ray free-electron laser facility, Phys. Rev. ST Accel. Beams 17, 080702 (2014).

[3] G. d'Auria et al., The compactlight design study project, in Proceedings of 10th International Particle Accelator Conference (IPAC 19), Melbourne, Australia (JACoW, Geneva, 2019).

[4] M. Diomede, D. Alesini, M. Bellaveglia et al., Preliminary rf design of an x-band linac for the eupraxia@ sparc_lab project, Nucl. Instrum. Methods Phys. Res., Sect. A 909, 243 (2018).

[5] T. Schietinger, M. Pedrozzi, M. Aiba et al., Commissioning experience and beam physics measurements at the swissfel injector test facility, Phys. Rev. Accel. Beams 19, 100702 (2016).
[6] I. Abe, N. Akasaka, M. Akemoto et al., The kekb injector linac, Nucl. Instrum. Methods Phys. Res., Sect. A 499, 167 (2003).

[7] C. Tang, W. Huang et al., Tsinghua thomson scattering X-ray source, Nucl. Instrum. Methods Phys. Res., Sect. A 608, S70 (2009).

[8] E. I. Simakov, V. A. Dolgashev, and S. G. Tantawi, Advances in high gradient normal conducting accelerator structures, Nucl. Instrum. Methods Phys. Res., Sect. A 907, 221 (2018).

[9] T. Higo, Progress of X-Band Accelerating Structures, in Proceedings of 25th International Linear Accelerator Conference (LINAC 10), 2010, Tsukuba, Japan (JACoW, Geneva, 2010), pp. 1038-1042.

[10] T. Higo, Y. Higashi, S. Matsumoto, K. Yokoyama, and J. W. Wang, Advances in X-band TW accelerator structures operating in the $100 \mathrm{mv} / \mathrm{m}$ regime, in Proceedings of $1 \mathrm{st}$ International Particle Accelerator Conference (IPAC 10), Kyoto, China (JACoW, Geneva, 2010), pp. 3702-3704.

[11] J. Wang, J. Lewandowski, J. Pelt, C. Yoneda, and S. Menlo, Fabrication technologies of the high gradient accelerator structures at $100 \mathrm{mv} / \mathrm{m}$ range, in Proceedings of $1 \mathrm{st}$ International Particle Accelerator Conference (IPAC 10), Kyoto, China (2010), pp. 3849-3821.

[12] R. H. Fowler and L. Nordheim, Electron emission in intense electric fields, Proc. R. Soc. A 119, 173 (1928).

[13] P. Wilson, Z. Farkas, and R. Ruth, Field emission and rf breakdown in high-gradient room-temperature linac structure, Stanford Linear Accelerator Center, Menlo Park, CA (USA), Technical Report No. SLAC-PUB-7684, 1997.

[14] J. Shao, S. P. Antipov, S. V. Baryshev, H. Chen, C. Manoel, D. S. Doran et al., Observation of Field-Emission Dependence on Stored Energy, Phys. Rev. Lett. 115, 264802 (2015).

[15] X. Wu, J. Shi, H. Chen, J. Shao, T. Abe, T. Higo, S. Matsumoto, and W. Wuensch, High-gradient breakdown studies of an $x$-band compact linear collider prototype structure, Phys. Rev. Accel. Beams 20, 052001 (2017).

[16] A. Degiovanni, W. Wuensch, and J. G. Navarro, Comparison of the conditioning of high gradient accelerating structures, Phys. Rev. Accel. Beams 19, 032001 (2016).

[17] A. Grudiev, S. Calatroni, and W. Wuensch, New local field quantity describing the high gradient limit of accelerating structures, Phys. Rev. ST Accel. Beams 12, 102001 (2009).

[18] T. Higo, T. Abe, Y. Arakida et al., Comparison of high gradient performance in varying cavity geometries, in Proceedings of 4th International Particle Accelerator Conference (IPAC 13), Shanghai, China (2013), pp. 2741-2743.

[19] J. Rodriguez, G. Arnau-Izquierdo, R. Corsini, S. Dobert, R. Fandos, A. Grudiev, I. Syratchev, M. Taborelli, F. Tecker, P. Urschutz, W. Wuensch, O. Mete, H. Aksakal, Z. Nergiz, and $\mathrm{M}$. Johnson, $30 \mathrm{GHz}$ high-gradient accelerating structure test results, in 2007 IEEE Particle Accelerator Conference (PAC) (2007), pp. 3818-3820.

[20] H. Braun, S. Dobert, L. Groening, I. Wilson, and W. Wuensch, Status of CLIC high-gradient studies, in PACS2001. Proceedings of the 2001 Particle Accelerator Conference (2001) (Cat. No. 01CH37268) (IEEE, New York, 2001), Vol. 2, pp. 852-854. 
[21] H. H. Braun, S. Döbert, I. Wilson, and W. Wuensch, Frequency and Temperature Dependence of Electrical Breakdown at 21, 30, and $39 \mathrm{GHz}$, Phys. Rev. Lett. 90, 224801 (2003).

[22] J. Power, in 13th International Workshop on Breakdown Science and High-Gradient Technology (HG 2021), https://indico.fnal.gov/event/22025/contributions/210368/ attachments/142268/179578/JohnPower-AWA-HG21.pdf.

[23] N. A. Solyak, Gradient limitations in room temperature and superconducting acceleration structures, AIP Conf. Proc. 1086, 365 (2009).

[24] V. Dolgashev, S. Tantawi, and Y. Higashi, and T. Higo, Status of high power tests of normal conducting single-cell structures, in Proceedings of 11th European Particle Accelerator Conference (EPAC 08), Genoa, Italy (2008), pp. 742-744.

[25] R. M. Sundelin, J. L. Kirchgessner, and M. Tigner, Parallel coupled cavity structure, IEEE Trans. Nucl. Sci. 24, 1686 (1977).

[26] O. Brezhnev, P. Logatchev, V. Pavlov, O. Pirogov, S. Shiyankov, J. Chernousov, V. Ivannikov, and I. Shebolaev, Parallel-coupled accelerating structures, in $21 \mathrm{st}$ International Linear Accelerator Conference (LINAC 02), Gyeongju, Korea (2002), pp. 213-215.

[27] J. Neilson, S. Tantawi, and V. Dolgashev, Design of rf feed system and cavities for standing-wave accelerator structure, Nucl. Instrum. Methods Phys. Res., Sect. A 657, 52 (2011).

[28] S. Tantawi, M. Nasr, Z. Li, C. Limborg, and P. Borchard, Design and demonstration of a distributed-coupling linear accelerator structure, Phys. Rev. Accel. Beams 23, 092001 (2020).

[29] S. V. Kutsaev, Advanced technologies for applied particle accelerators and examples of their use (review), Tech. Phys. 66, 161 (2021).

[30] J. Gao, Analytical approach and scaling laws in the design of disk-loaded travelling wave accelerating structures, Part. Accel. 43, 235 (1994), http://cds.cern.ch/record/ 1108283/files/p235.pdf.
[31] V. A. Dolgashev, S. G. Tantawi, A. D. Yeremian, Z. Li, and B. Higashi, Y. Spataro, Status of high power tests of normal conducting short standing wave structures, in Proceedings of 2nd International Particle Acceler Conference (IPAC 11), San Sebastian, Spain (2011), pp. 241-243.

[32] T. Argyropoulos, N. Catalan-Lasheras, A. Grudiev, G. Mcmonagle, E. Rodriguez-Castro, I. Syrachev et al., Design, fabrication, and high-gradient testing of an $x$-band, traveling-wave accelerating structure milled from copper halves, Phys. Rev. Accel. Beams 21, 061001 (2018).

[33] CST studio suite, Computer Simulation Technology (CST).

[34] Z. Farkas, H. Hogg, G. Loew, and P. Wilson, Sled: A method of doubling slac's energy, in Proceedings of 9th International Conference on High Energy Accelerators, Stanford (SLAC, California, 1974), p. 576.

[35] J. Wang, S. Tantawi, C. Xu, M. Franzi, P. Krejcik, G. Bowden et al., Development for a supercompact $x$-band pulse compression system and its application at SLAC, Phys. Rev. Accel. Beams 20, 110401 (2017).

[36] B. Woolley, I. Syratchev, and A. Dexter, Control and performance improvements of a pulse compressor in use for testing accelerating structures at high power, Phys. Rev. Accel. Beams 20, 101001 (2017).

[37] P. Craievich, M. Bopp, H. Braun, A. Citterio, R. Fortunati, R. Ganter et al., Novel $x$-band transverse deflection structure with variable polarization, Phys. Rev. Accel. Beams 23, 112001 (2020).

[38] Y. Jiang, H. Zha, J. Shi, M. Peng, X. Lin, and H. Chen, A compact $\mathrm{x}$-band microwave pulse compressor using a corrugated cylindrical cavity, IEEE Trans. Microwave Theory Tech. 69, 1586 (2021).

[39] Y. Jiang, J. Shi, P. Wang, H. Zha, X. Lin, F. Liu, C. Cheng, and $\mathrm{H}$. Chen, Compact two-stage pulse compression system for producing gigawatt microwave pulses, IEEE Trans. Microwave Theory Tech. 69, 4533 (2021). 